and emaciation; chest organs normal. Specific treatment was at once adopted - for the first few weeks iodide of potassium alone, then in combination with grey powder,with little benefit; corrosive chloride and other inhalations were likewise used. In about three months' time the left wall of the larynx had become invaded by a pathological deposit effacing the local band of the same side, and fixing the left arytenoid cartilage. In another two months this deposit had extended to the right side of the larynx, causing destruction of the same vocal cord in a similar fashion, without, however, implicating the right arytenoid cartilage.

The patient was then lost sight of for six months. On returning, examination showed the larynx on each side up to the level of the false cords to be the seat of fungating growths, that of the left side being the largest. The patient had by this time become markedly emaciated and voiceless. The cough was incessant at night, and swallowing gave pain. The left arytenoid was now fixed, abducted, and depressed. A variety of remedies were applied locally to the larynx-e.g., lactic acid, menthol, and (once) chromic acid, - but without effect. The galvano-cautery was once used, but with no good result. The chink left for respiration between the two fungating masses in the larynx becoming almost daily smaller, tracheotomy was suggested to the patient, but was declined. Chloral and paregoric were used as sedatives for the cough during the interval. One month before operation a phlegmonous swelling with pain began over the left side of the larynx, which rapidly increased in its dimensions, and, extending from the left angle of the jaw (and fixing the same) down to the middle of the neck and round the front of the larynx, obscured all its landmarks. The patient's condition with this became much more serious, respiration and swallowing being both alike difficult.

Consent was now obtained for operation. After chloroform had been given, a straight incision, as that for tracheotomy, was made along the middle line as far as that could be made out, and on penetrating the deep layer of cervical fascia, which was clearly seen, a large escape (two ounces) of foul-smelling pus took place from a considerable cavity, extending the length of the larynx on the left side. Furthe operative procedure was postponed until this cavity should be effectually treated and closed, more especially as it was noticed that, as the pus escaped, the breathing became easier. It was, besides, thought that, were the trachea to be opened at once, disaster might follow the trickling of any of this foul pus into the lungs.

All trace of the abscess cavity and swelling had disappeared in the course of ten days. No necrosis of cartilage could be made out as a cause for the suppuration, nor was there any sinus found leading from the abscess. The patient was accordingly once more put under chloroform, the first incision extended downward through the isthmus (which gave little trouble and was tied on either side), and the tube inserted through an incision in the upper three rings of the trachea.

For four days after operation the patient suffered severely from cough, difficulty in swallowing, and stiffness of the jaws; but since then, up to the present date, he has enjoyed fair sleep at night, freedom from cough, and a good appetite. His appearance has improved, and he has put on flesh. Once or twice fetor of breath has been noticed, and a slight staining with blood of the discharge from the mouth. Examination of neoplasn in the larynx shows that that of the left side is enlarged and overlaps that of the right, thus almost closing the glottis. The musculature of the right side of the larynx is still free, and, when acting, a craterlike cavity is seen, lined with granulations. The growth extends quite up to the line of false band, the upper surface of which on each side as yet is only infiltrated and inflamed. The external configuration of the larynx is normal, with very little enlargement of the cervical glands.

Remarlis. - The affection began, as was clearly seen, in the left local band (or closely in its neighbourhood), rendering it pale, flaccid, and immobile; that to this succeeded ulceration with disappearance of cord, and, later, on granulations; that the same course was observed on the right side; that fully twelve months passed before ulceration was established and sprouting commenced. The abscess that formed at the side of the larynx is probably to be accounted for by putrid absorption from perichondritis. Fully five months has now elapsed since the tracheotomy was performed, and so far the patient is in a much better condition than he was for months before the operation.

Newcastle-on-Tyne.

\section{KEHRER'S OPERATION FOR DEPRESSED NIPPLE.}

BY G. ERNEST HERMAN, M.B. LoND., F.R.C.P., OBSTETRIC PIIYSICIAN TO TIIE LONDON IIOSPITAL, ETC.

Professor 1. A. Kenner of Heidelberg has suggested ${ }^{x}$ and practised an operation for the cure of depressed nipple. The cases in which this operation is indicated are those in which the nipple lies in a sort of cup, so that it does not project enough above the surface for the child to seize it, but is otherwise well formed, as in Fig. 1. The operation consists in the excision of a ring of skin, or of two crescentic pieces of skin, surrounding the nipple; so that in the healing of the denuded surface the approximation of the internal and external edges of the ing or crescent may so pull upon the skin immediately surrounding the nipple, and forming the cup in which it lies, as to obliterate this cup and render the nipple accessible to the child.

So far as I am aware, this small, but I think useful

Frg. 1.

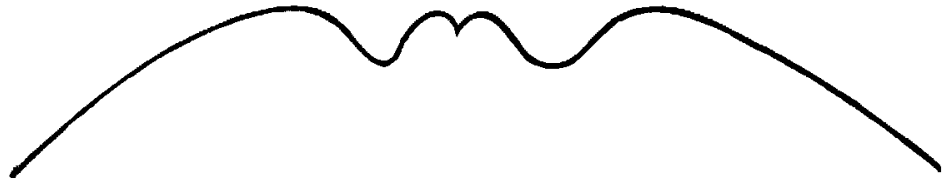

operation, has not yet attracted attention in this country. I have performed it twice. One of the cases I have been unable to keep under observation. In the other I am now able to judge of the effect of the operation.

The patient, aged thirty, has had three children. The first child she suckled with the left breast, the nipple of which was well-shaped and prominent, but not with the right. The right nipple was sunk in a hollow, so that the child could not seize it, and its apex was drawn in so as to form a dimple (Fig. 1). In May, 1887, she was delivered of her second child in the General Lying-in Hospital. She suckled, as before, with the left breast, but the child was unable to take the right. Five days after delivery I performed Kehrer's operation on the right breast. As tlie dimple on the nipple ran horizontally, I removed two crescent-shaped pieces of skin, one above the other below the nipple, each piece measuring about two inches long by about an inch across its wirlest part (see Fig. 2). The patient returned to the hospital to be delivered of her third child in May, 1889.

She can now suckle with both breasts. The right nipple, although not so prominent or well-shaped as the left, yet projects enough for the child to grasp it. The object of the operation has therefore been attained.

Hasley-street, $W$

\section{A MALLEABLE TRUSS FOR CORRECTING DEFORMITIES OF THE NOSE.}

BY W. J. WALSHAM, F.R.C.S.,

ASSISTANT SUIRGEON TO ST. BARTHOLOMEW'S HOSPITAL, AND SURGEON IN CHARGE OF THE ORTHOPADIC DEPARTMENT.

IN THE LANCET of Sept. 20th, 1884, I described a nasal mask for gaining a fixed point whereon to secure the nasal truss ; and, again, in the number of Feb. 25th, 1888, a poroplastic cap for the same purpose. I have now to describe a new form of truss. In the older contrivances the correction 